\title{
COMPLEMENTS TO SOLVABLE HALL SUBGROUPS ${ }^{1}$
}

\author{
ROBERT GILMAN
}

ABSTRACT. A Hall subgroup $H$ of a finite group $G$ is a subgroup whose order is relatively prime to its index. We show that if $H$ is solvable and if the way prime power elements of $H$ are conjugate in $G$ is restricted, then $G$ has a quotient isomorphic to $H$.

Suppose $H$ is a Hall subgroup of $G$. Let $\pi$ be the set of prime divisors of the order of $H$. A number is $\pi$ if all its prime divisors are in $\pi$ and $\pi^{\prime}$ if none of them are. The index $(G: H)$ is $\pi^{\prime}$. If $H$ has a normal complement in $G$, then any two elements of $H$ conjugate in $G$ are conjugate by an element of $H$; i.e. $H$ is $c$-closed. Taking $H$ to be the permutation group on four letters and $G$ to be the one on five letters shows that a $c$-closed solvable Hall subgroup need not have a normal complement. However if $H$ has a normal complement we also know that for any subgroup $D$ of $H$ the centralizer in $H$ of $D$ is a Hall subgroup of the centralizer of $D$. Thus we have

(1) If $H$ has a normal complement, then $H$ is $c$-closed and $\left(C(D): C_{H}(D)\right)$ is $\pi^{\prime}$.

If $H$ is a solvable Hall $\pi$ subgroup satisfying (1), then by Proposition $1, H$ has a normal complement. Propositions 2 and 3 give other sufficient conditions. From now on we assume $H$ is a solvable Hall $\pi$ subgroup of $G$.

Proposition 1. If for every $x \in G$ and prime power element $h \in H$ such that $h^{x}=x^{-1} h x \in H$ there exist elements $y_{1} \cdots y_{k}$ and subgroups $B_{1} \cdots B_{k}$ satisfying

(a) $x=y_{1} \cdots y_{k}$

(b) $y_{i} \in B_{i} H$, the set of products of elements from $B_{i}$ and $H$,

(c) $B_{1} \subset C(h), B_{i} \subset C\left(h^{y_{1} \cdots y_{i-1}}\right)$ for $2 \leqq i \leqq k$,

(d) $\left(B_{i}: H \cap B_{i}\right)$ is $\pi^{\prime}$,

then $H$ has a normal complement.

Proof. By Corollary 1 to Theorem 9 and the corollary to Theorem $10^{*}$ of $[2, \S 5]$ or by a transfer argument we can find $N$ normal in $G$ such that $H N=G, N \neq G$. Let $M=H \cap N, C_{i}=B_{i} \cap N$. We claim that

Received by the editors April 13, 1970.

AMS 1970 subject classifications. Primary 20D20; Secondary 20D10.

Key words and phrases. Solvable Hall subgroup, normal complement, finite group, quotient group, $c$-closed, transfer, prime power, fusion.

${ }^{1}$ Contains part of the author's dissertation. 
if $x \in N$, then (a) through (d) still hold if we substitute $M$ for $H$ and $C_{i}$ for $B_{i}$. It is easy to see that (a), (c) and (d) are valid; we prove (b) by induction on $k$. Since $(G: N)$ is $\pi$, we have by (d) $B_{i}=C_{i}\left(B_{i} \cap H\right)$ and $B_{i} H=C_{i} H$. If $k=1, y_{1} \in B_{1} H \cap N$ implies $y_{1} \in C_{1} M$. If $k \geqq 2$, let $z=y_{1} \cdots y_{k-1}$. By (b) and (c) $h^{z} \in H$, and so by induction $y_{i} \in C_{i} M$ $\subset N$. As before $y_{k}=z^{-1} x \in B_{k} H \cap N$ implies $y_{k} \in C_{k} M$.

If we restrict $k$ to be 1 , then Proposition 1 and (1) give the following corollary:

COROLlaRY 1. H has a normal complement iff

(a) any two prime power elements of $H$ which are conjugate in $G$ are conjugate in $H$,

(b) for any p.p. element $h \in H,\left(C(h): C_{H}(h)\right)$ is $\pi^{\prime}$.

We remark that if $H$ is not a solvable Hall subgroup of $G$, but $(G: H)$ is $\pi^{\prime}$ and the other hypotheses of Proposition 1 hold, then the proof yields that the maximal solvable $\pi$ quotients of $G$ and $H$ are isomorphic.

Corollary 2 (Brauer and Suzuki). H has a normal complement iff

(a) any two p.p. elements of $H$ which are conjugate in $G$ are conjugate in $H$,

(b) any subgroup $E$ which is the product of a cyclic $p$ group and a $q$ group for $p, q \in \pi$ has a conjugate in $H$.

Proof. We show that the hypotheses imply that (b) of Corollary 1 holds. Let $E$ be the direct product of the cyclic group generated by $h$ and a Sylow $q$ subgroup $Q$ of $C(h)$ for any $q \in \pi$.

By (b), $E^{x} \subset H$. Hence $h^{x} \subset H$ and $h^{x}=h^{k}$ for some $k \in H$ by (a). Taking $y=x k^{-1}$ we have $Q^{v} \subset E^{v} \subset H \cap C\left(h^{y}\right)=C_{H}(h)$. The converse follows from the Schur-Zassenhaus Theorem.

Let $H^{*}$ be the subgroup of $H$ generated by all intersections $H \cap H^{*}, x \notin N(H)$. If $H$ is normal in $G$ set $H^{*}=H$. Note that $N(H)$ $\subset N\left(H^{*}\right)$.

Proposition 2. Suppose $G=R S R$ for two subgroups $R$ and $S$ satisfying $N(H) \subset R \subset N\left(H^{*}\right), H \subset S$. If $H$ has a normal complement in $R$ and in $S$, then $H$ has one in $G$.

Proof. We apply Proposition 1.

Suppose $h, h^{x} \in H$. If $h \in H^{*}$, then $x \in R$ and by (1) we can take $y_{1}=x, B_{1}=C_{R}(h)$. Otherwise $x=r s t, r, t \in R, s \in S$ and $h^{r}, h^{r s} \in H$. In this case $y_{1}=r, y_{2}=s, y_{3}=t$ and $B_{1}=C_{R}(h), B_{2}=C_{S}\left(h^{r}\right), B_{3}=C_{R}\left(h^{r s}\right)$. 
Because Proposition 1 refers to conjugation of prime power elements, we can apply Alperin's results on fusion. From the definition of a conjugation family [1, Definitions $5.1,5.3]$ we obtain:

Proposition 3. For each $p \in \pi$ pick a Sylow $p$ subgroup $H_{p}$ of $H$ and a conjugation family for $H_{p}$. Let $F=\left\{\left(D_{i}, T_{i}\right)\right\}$ be the union of these families and the set $\left\{\left(H_{p}, N\left(H_{p}\right)\right)\right\}$. If for each $\left(D_{i}, T_{i}\right) \in F$ and $h \in D_{i}$ there exists a subgroup $B_{i}$ satisfying $T_{i} \subset B_{i} H, B_{i} \subset C(h)$, $\left(B_{i}: H \cap B_{i}\right)$ is $\pi^{\prime}$, then $H$ has a normal complement.

In particular for the conjugation family of [1, Theorem 5.1] we have the following result:

COROLlARY 1. If for each $p \in \pi$ and $p$ subgroup $D \subset H_{p}$ we have

(a) $\left(C(D): C_{H}(D)\right)$ is $\pi^{\prime}$,

(b) $N(D)=C(D) N_{H}(D)$ if $C_{H_{p}}(D) \nsubseteq D$,

then $H$ has a normal complement.

\section{BiBLIOGRAPHY}

1. J. L. Alperin, Sylow intersections and fusion, J. Algebra 6 (1967), 222-241. MR $35 \# 6748$.

2. R. Brauer, A characterization of the characters of groups of finite order, Ann. of Math. (2) 57 (1953), 357-377. MR 14, 844.

3. - On quotient groups of finite groups, Math. Z. 83 (1964), 72-84. MR 28 \#3088.

4. M. Suzuki, On the existence of a Hall normal subgroup, J. Math. Soc. Japan 15 (1963), 387-391. MR 28 \#2157.

Stevens Institute of Technology, Hoboken, New Jersey 07030 